\title{
Stability Analysis of a Multigroup SEIR Epidemic Model with General Latency Distributions
}

\author{
Nan Wang, Jingmei Pang, and Jinliang Wang \\ School of Mathematical Science, Heilongjiang University, Harbin 150080, China \\ Correspondence should be addressed to Jinliang Wang; jinliangwang@aliyun.com \\ Received 11 February 2014; Accepted 27 March 2014; Published 17 April 2014 \\ Academic Editor: Kaifa Wang
}

Copyright ( 2014 Nan Wang et al. This is an open access article distributed under the Creative Commons Attribution License, which permits unrestricted use, distribution, and reproduction in any medium, provided the original work is properly cited.

\begin{abstract}
The global stability of a multigroup SEIR epidemic model with general latency distribution and general incidence rate is investigated. Under the given assumptions, the basic reproduction number $\Re_{0}$ is defined and proved as the role of a threshold; that is, the diseasefree equilibrium $P_{0}$ is globally asymptotically stable if $\mathfrak{R}_{0} \leq 1$, while an endemic equilibrium $P^{*}$ exists uniquely and is globally asymptotically stable if $\Re_{0}>1$. For the proofs, we apply the classical method of Lyapunov functionals and a recently developed graph-theoretic approach.
\end{abstract}

\section{Introduction}

Mathematical models have become important tools in analyzing the spread and control of infectious diseases. The SIR model is one of the most popular ones in this field, for which the total population is subdivided into three compartments: susceptible, infectious, and removed. For some diseases, it is reasonable to include a latent (or exposed) class for those susceptible individuals who are infected with the disease but are not yet infectious, which leads to SEIR model [1-6]. Let $S(t), E(t), I(t)$, and $R(t)$ be the numbers of individuals in the susceptible, exposed, infectious, and removed compartments, respectively, with the total population $N(t)=S(t)+E(t)+$ $I(t)+R(t)$. Suppose that $d>0$ represents the constant recruitment rate and the natural mortality rate. Assuming mass action for the disease transmission and letting $\beta>0$ denote the effective contact rate, the rate of change of $S(t)$ is

$$
S^{\prime}(t)=d-\beta S(t) I(t)-d S(t) .
$$

Taking into consideration a general exposed distribution, van den Driessche et al. [5] formulated and studied the following model:

$$
\begin{aligned}
& S^{\prime}(t)=d-\beta S(t) I(t)-d S(t), \\
& E(t)=\int_{0}^{t} \beta S(u) I(u) e^{-d(t-u)} P(t-u) \mathrm{d} u,
\end{aligned}
$$

$$
\begin{aligned}
R^{\prime}(t) & =r I(t)-d R(t), \\
I(t) & =N-S(t)-E(t)-R(t),
\end{aligned}
$$

where $r \geq 0$ is the rate at which infective individuals recover. $N$ is constant total populations. It is assumed in [5] that individuals rarely die of the disease and the disease-induced death is negligible, which ensures a constant population; that is, $N(t)=N \cdot P(t)$ denotes the probability (without taking death into account) that an exposed individual still remains in the exposed class $t$ time units after entering the exposed class and it satisfies the following.

$\left(\mathbf{A}_{1}\right) P:[0, \infty) \rightarrow[0,1]$ is nonincreasing, piecewise continuous with possibly finitely many jumps and satisfies $P\left(0_{+}\right)=1, \lim _{t \rightarrow \infty} P(t)=0$ with $\int_{0}^{\infty} P(u) d u$ being positive and finite.

In fact, the integral term in model (2) is in the sense of Riemann-Stieltjes integrals; the second equation of (2) takes the following form:

$$
\begin{aligned}
E^{\prime}(t)= & \beta S(t) I(t)-d E(t) \\
& +\int_{0}^{t} \beta S(u) I(u) e^{-d(t-u)} d_{t} P(t-u) \mathrm{d} u,
\end{aligned}
$$


where $d_{t} P(t-u)=d P(t-u) / d t$. It follows from total population size $N$ which is constant that the rate of change of $I$ is governed by

$$
I^{\prime}(t)=-\int_{0}^{t} \beta S(u) I(u) e^{-d(t-u)} d_{t} P(t-u) \mathrm{d} u-(d+r) I(t) .
$$

Thus, model (2) can be written as the system

$$
\begin{aligned}
S^{\prime}(t)= & d-\beta S(t) I(t)-d S(t), \\
E^{\prime}(t)= & \beta S(t) I(t)-d E(t) \\
& +\int_{0}^{t} \beta S(u) I(u) e^{-d(t-u)} d_{t} P(t-u) \mathrm{d} u, \\
I^{\prime}(t)= & -\int_{0}^{t} \beta S(u) I(u) e^{-d(t-u)} d_{t} P(t-u) \mathrm{d} u \\
& -(d+r) I(t), \\
R^{\prime}(t)= & r I(t)-d R(t) .
\end{aligned}
$$

Recently, a model of this type including the possibility of disease relapse has been proposed in $[5,6]$ to study the transmission and spread of some infectious diseases such as herpes, and its global dynamics have been completely investigated in $[5,7]$.

Heterogeneity in the host population can result from different contact modes such as those among children and adults for childhood diseases (e.g., measles and mumps) or different behaviors such as the numbers of sexual partners for some sexually transmitted infections (e.g., herpes and condyloma acuminatum). Taking into consideration different contact patterns, distinct number of sexual partners, or different geography and so forth, it is more proper to divide individual hosts into groups. Therefore, lots of multigroup models have been proposed in the literature to describe the transmission of infectious disease in heterogeneity environment (see [8-17] and references cited therein).

In multigroup epidemic models, a heterogeneous host population is divided into several homogeneous groups according to modes of transmission, contact patterns, or geographic distributions, so that within-group and intergroup interactions can be modeled separately. In this paper, we formulate a multigroup SEIR epidemic model with general exposed distribution and general incidence rates. The population is divided into $n$ distinct groups $(n \geq 2)$. For $1 \leq$ $k \leq n$, the $k$ th group is further partitioned into four compartments: susceptible, exposed, infectious, and recovered, whose numbers of individuals at time $t$ are denoted by $S_{k}(t), E_{k}(t)$, $I_{k}(t)$, and $R_{k}(t)$, respectively. Within the $k$ th group, $\varphi_{k}\left(S_{k}\right)$ represents the growth rate of $S_{k}$, which includes both the production and the natural death of susceptible individuals.

In [18], Zhang et al. studied a multigroup SEIR epidemic model with general exposed distribution and general incidence rates. By using the well-known "linear chain trick," the authors reformulate the model into an equivalent ordinary differential equations system. The global stability results of equilibria are obtained by constructing suitable Lyapunov functionals for general incidence rate function $f_{k j}\left(S_{k}(t), I_{j}(t)\right)$. In [19], Hattaf et al. introduced a general incidence rate $f(S, I) I$ in a delayed SIR epidemic model.

Motivated by these facts, in this paper, we incorporate the general incidence rate presented in [19] to the following system of differential and integral equations:

$$
S_{k}^{\prime}(t)=\varphi_{k}\left(S_{k}(t)\right)-\sum_{j=1}^{n} f_{k j}\left(S_{k}(t), I_{j}(t)\right) I_{j}(t),
$$

$$
\begin{aligned}
& E_{k}^{\prime}(t) \\
& \begin{aligned}
= & \sum_{j=1}^{n} f_{k j}\left(S_{k}(t), I_{j}(t)\right) I_{j}(t) \\
& -\sum_{j=1}^{n} \int_{0}^{t} f_{k j}\left(S_{k}(u), I_{j}(u)\right) I_{j}(u) e^{-\delta_{k}(t-u)} g_{k}(t-u) \mathrm{d} u \\
& -\delta_{k} E_{k}(t), \\
I_{k}^{\prime}(t)= & \sum_{j=1}^{n} \int_{0}^{t} f_{k j}\left(S_{k}(u), I_{j}(u)\right) I_{j}(u) e^{-\delta_{k}(t-u)} g_{k}(t-u) \mathrm{d} u \\
\quad & -\left(\delta_{k}+\gamma_{k}\right) I_{k}(t), \\
& R_{k}^{\prime}(t)=\gamma_{k} I_{k}(t)-\delta_{k} R_{k}(t),
\end{aligned}
\end{aligned}
$$

where $g_{j}(t)=-P_{j}^{\prime}(t)$, the nonlinear term $f_{k j}\left(S_{k}(t), I_{j}(t)\right) I_{j}(t)$ represents the cross-infection from group $j$ to group $k, \delta_{k}$ denotes the natural death rates of exposed and infectious classes in the $k$ th group, and $\gamma_{k}$ denotes the production of the recovered individuals from infectious ones in the $k$ th group. All constants $\delta_{k}, \gamma_{k}, k=1,2, \ldots, n$, are assumed to be positive.

The organization of this paper is as follows; in the next section, we give some preliminaries of our main model. In Section 3, we prove the global asymptotic stability of the disease-free equilibrium $P_{0}$ for $\mathfrak{R}_{0} \leq 1$ using the classical method of Lyapunov. The existence of endemic equilibrium is also proved. In Section 4, we prove global asymptotic stability of an endemic equilibrium $P^{*}$ for $\Re_{0}>1$ using the graphtheoretic approach.

\section{Preliminaries}

Since the variables $E_{k}$ and $R_{k}$ do not appear in the first and third equations of (6), we can only consider the reduced system as follows:

$$
\begin{gathered}
S_{k}^{\prime}(t)=\varphi_{k}\left(S_{k}(t)\right)-\sum_{j=1}^{n} f_{k j}\left(S_{k}(t), I_{j}(t)\right) I_{j}(t), \\
I_{k}^{\prime}(t)=\sum_{j=1}^{n} \int_{0}^{t} f_{k j}\left(S_{k}(u), I_{j}(u)\right) I_{j}(u) e^{-\delta_{k}(t-u)} g_{k}(t-u) \mathrm{d} u \\
-\left(\delta_{k}+\gamma_{k}\right) I_{k}(t) .
\end{gathered}
$$


The incidence function $f_{k j}\left(S_{k}, I_{j}\right)$ in (7) is assumed to be continuously differentiable in the interior of $\mathbb{R}_{+}^{2}$ and to satisfy the following hypotheses:

$\left(S_{1}\right) f_{k j}\left(0, I_{j}\right)=0$, for all $I_{j} \geq 0$;

$\left(S_{2}\right) \partial f_{k j}\left(S_{k}, I_{j}\right) / \partial S_{k}>0$, for all $S_{k}>0$ and $I_{j} \geq 0$;

$\left(S_{3}\right) \partial f_{k j}\left(S_{k}, I_{j}\right) / \partial I_{j} \leq 0$, for all $S_{k} \geq 0$ and $I_{j} \geq 0$;

assume that the functions $\varphi_{k}$ satisfy the following conditions:

$\left(\mathrm{S}_{4}\right) \varphi_{k}$ are local Lipschitz on $[0, \infty)$ with $\varphi_{k}(0)>0$, and there is a unique positive solution $\xi=S_{k}^{0}$ for the equation $\varphi_{k}(\xi)=0 ; \varphi_{k}\left(S_{k}\right)>0$ for $0 \leq S_{k}<S_{k}^{0}$, and $\varphi_{k}\left(S_{k}\right)<0$ for $S_{k}>S_{k}^{0}$.

Typical examples of $f_{k j}\left(S_{k}, I_{j}\right)$ satisfying $\left(\mathrm{S}_{1}\right)-\left(\mathrm{S}_{3}\right)$ include common incidence functions such as

$$
\begin{gathered}
f_{k j}\left(S_{k}, I_{j}\right)=S_{k} I_{j}[20,2,3], \quad f_{k j}\left(S_{k}, I_{j}\right)=S_{k}^{q} I_{j}[21], \\
f_{k j}\left(S_{k}, I_{j}\right)=\frac{\eta S_{k} I_{j}}{1+\theta S_{k}}[1] .
\end{gathered}
$$

The class of $\varphi_{k}\left(S_{k}\right)$ that satisfies $\left(S_{4}\right)$ includes both $\lambda_{k}-d_{k}^{S} S_{k}$ and $\lambda_{k}-d_{k}^{S} S_{k}+r_{k} S_{k}\left(1-S_{k} / N_{k}\right)$, which have been widely used in the literature of population dynamics $[1,8]$.

For model (7), the existence, uniqueness, and continuity of solutions follow from the theory for integrodifferential equations in [22]. It can be easily verified that every solution of (7) with nonnegative initial conditions remains nonnegative. It follows from $\left(S_{4}\right)$ and the first equation in (7) that $S_{k}^{\prime}(t) \leq \varphi_{k}\left(S_{k}(t)\right)$, and thus

$$
\limsup _{t \rightarrow \infty} S_{k}(t) \leq S_{k}^{0}, \quad \text { for } 1 \leq k \leq n .
$$

From the biological significance, we only need to consider (7) in the following region:

$$
\begin{aligned}
\Gamma:= & \left\{\left(S_{1}, I_{1}, S_{2}, I_{2}, \ldots, S_{n}, I_{n}\right)\right. \\
& \left.\in \mathbb{R}_{+}^{2 n}: S_{k}, I_{k} \geq 0, S_{k}+I_{k} \leq S_{k}^{0}, 1 \leq k \leq n\right\} .
\end{aligned}
$$

Indeed, one can easily verify that the set $\Gamma$ is positively invariant with respect to (7).

It is clear that system (7) has a disease-free equilibrium $P_{0}=\left(S_{1}^{0}, 0, S_{1}^{0}, 0, \ldots, S_{n}^{0}, 0\right)$ in $\Gamma$. Next, we will give some notations which will be useful for our main results.

Let

$$
\begin{aligned}
J(\xi) & =\int_{\xi}^{\infty} g_{k}(u) e^{-\delta_{k} u} \mathrm{~d} u, \\
Q_{k} & =J(0)=\int_{0}^{\infty} g_{k}(u) e^{-\delta_{k} u} \mathrm{~d} u .
\end{aligned}
$$

It can be verified that $Q_{k} \in(0,1)$.
For finite time $t$, system (7) may not have an endemic equilibrium. If system (7) has an endemic equilibrium, the endemic equilibrium must satisfy the limiting system

$$
\begin{gathered}
S_{k}^{\prime}(t)=\varphi_{k}\left(S_{k}(t)\right)-\sum_{j=1}^{n} f_{k j}\left(S_{k}(t), I_{j}(t)\right) I_{j}(t), \\
I_{k}^{\prime}(t)=\sum_{j=1}^{n} \int_{0}^{\infty} f_{k j}\left(S_{k}(t-u), I_{j}(t-u)\right) \\
\times I_{j}(t-u) e^{-\delta_{k} u} g_{k}(u) \mathrm{d} u \\
-\left(\delta_{k}+\gamma_{k}\right) I_{k}(t) .
\end{gathered}
$$

Since the limiting system (12) contains an infinite delay, its associated initial condition needs to be restricted in an appropriate fading memory space. For any $\sigma_{k} \in\left(0, \delta_{k}\right)$, define the following Banach space of fading memory type (see $[23,24]$ and references therein):

$$
C_{k}=\left\{\phi_{k} \in C((-\infty, 0], \mathbb{R}): \phi_{k}(s) e^{\sigma_{k} s}\right.
$$

is uniformly continuous on $(-\infty, 0]$,

$$
\begin{aligned}
& \left.\sup _{s \leq 0}\left|\phi_{k}(s)\right| e^{\sigma_{k} s}<\infty\right\}, \\
& Y_{\Delta}=\left\{\phi_{k} \in C_{k}: \phi_{k}(s) \geq 0 \quad \forall s \leq 0\right\}
\end{aligned}
$$

with norm $\|\phi\|_{k}=\sup _{s \leq 0}|\phi(s)| e^{\sigma_{k} s}$. Let $\psi_{t} \in C_{i}$ and $t>0$ be such that $\psi_{t}(s)=\psi(t+s), s \in(-\infty, 0]$.

Let $\phi_{k}, \psi_{k} \in C_{k}$ such that $\phi_{k}(s), \psi_{k}(s) \geq 0$ for all $s \in(-\infty, 0]$. We consider solutions of system (12), $\left(S_{1 t}, I_{1 t}, \ldots, S_{n t}, I_{n t}\right)$, with initial conditions

$$
\left(\phi_{1}, \psi_{1}, \phi_{2}, \psi_{2}, \ldots, \phi_{n}, \psi_{n}\right) \text {. }
$$

The standard theory of functional differential equations [24] implies $\left(S_{1 t}, I_{1 t}, \ldots, S_{n t}, I_{n t}\right) \in C_{k}$ for all $t>0$. We study system (12) in the following phase space:

$$
\mathbb{X}_{\mathfrak{g}}=\prod_{k=1}^{n}\left(\mathbb{R} \times C_{k}\right) .
$$

It can be verified that solutions of (12) in $\mathbb{X}_{\mathfrak{g}}$ with initial conditions (14) remain nonnegative.

An equilibrium $P^{*}=\left(S_{1}^{*}, I_{1}^{*}, S_{2}^{*}, I_{2}^{*}, \ldots, S_{n}^{*}, I_{n}^{*}\right)$ in the interior of $\Gamma$ is called an endemic equilibrium of system (12), where $S_{k}^{*}, I_{k}^{*}>0$ satisfy the equilibrium equations

$$
\begin{gathered}
\varphi_{k}\left(S_{k}^{*}\right)=\sum_{j=1}^{n} f_{k j}\left(S_{k}^{*}, I_{j}^{*}\right) I_{j}^{*}, \\
\sum_{j=1}^{n} f_{k j}\left(S_{k}^{*}, I_{j}^{*}\right) I_{j}^{*} Q_{k}=\left(\delta_{k}+\gamma_{k}\right) I_{k}^{*} .
\end{gathered}
$$

Set $R_{0}=\rho\left(M^{0}\right)$ to denote the special radius of the matrix $M^{0}$, where

$$
M^{0}=\left(\frac{f_{k j}\left(S_{k}^{0}, 0\right) Q_{k}}{\delta_{k}+\gamma_{k}}\right)_{n \times n} .
$$


The parameter $R_{0}$ is defined as the basic reproduction number $[25,26]$. Since it can be verified that system (7) satisfies conditions $\left(A_{1}\right)-\left(A_{5}\right)$ of Theorem 2 of [26], we have the following lemma.

Lemma 1. For system (7), the disease-free equilibrium $P_{0}$ is locally asymptotically stable if $\Re_{0}<1$ while it is unstable if $\Re_{0}>1$.

\section{Global Stability of the Disease-Free Equilibrium}

Theorem 2. Assume that the functions $\varphi_{k}$ and $f_{k j}$ satisfy $\left(S_{1}\right)-\left(S_{4}\right)$, and $M^{0}$ is irreducible.

(i) If $\Re_{0} \leq 1$, then $P_{0}$ is the unique equilibrium of system (7), and $P_{0}$ is globally asymptotically stable in $\Gamma$.

(ii) If $\mathfrak{R}_{0}>1$, then $P_{0}$ is unstable and system (7) is uniformly persistent.

Proof. It follows from the Perron-Frobenius theorem (see Theorem 2.1.4 in [27]) that the nonnegative irreducible matrix $M^{0}$ has a positive eigenvector $\left(\omega_{1}, \omega_{2}, \ldots, \omega_{n}\right)$ such that

$$
\left(\omega_{1}, \omega_{2}, \ldots, \omega_{n}\right) \rho\left(M^{0}\right)=\left(\omega_{1}, \omega_{2}, \ldots, \omega_{n}\right) M^{0} .
$$

Now, we construct a Lyapunov functional

$$
V_{P_{0}}=\sum_{k=1}^{n} \frac{\omega_{k}}{\delta_{k}+\gamma_{k}} I_{k}
$$

Differentiating $V_{P_{0}}$ along the solution of system (7) and under $\left(\mathrm{S}_{2}\right)$ and $\left(\mathrm{S}_{3}\right)$, we obtain

$$
\begin{aligned}
& V_{P_{0}}^{\prime}=\sum_{k=1}^{n} \omega_{k}\left[\frac{1}{\delta_{k}+\gamma_{k}}\right. \times \sum_{j=1}^{n} \int_{0}^{t} f_{k j}\left(S_{k}(u), I_{j}(u)\right) I_{j}(u) \\
& \times e^{-\delta_{k}(t-u)} g_{k}(t-u) \mathrm{d} u \\
&\left.\quad-I_{k}(t)\right] \\
& \leq \sum_{k=1}^{n} \omega_{k}\left[\frac{1}{\delta_{k}+\gamma_{k}} \sum_{j=1}^{n} f_{k j}\left(S_{k}, 0\right) I_{j}(t) Q_{k}-I_{k}(t)\right] \\
& \leq \sum_{k=1}^{n} \omega_{k}\left[\frac{1}{\delta_{k}+\gamma_{k}} \sum_{j=1}^{n} f_{k j}\left(S_{k}^{0}, 0\right) I_{j}(t) Q_{k}-I_{k}(t)\right] \\
&=\left(\omega_{1}, \omega_{2}, \ldots, \omega_{n}\right)\left[M^{0} I-I\right] \\
&=\left[\rho\left(M^{0}\right)-1\right]\left(\omega_{1}, \omega_{2}, \ldots, \omega_{n}\right) I,
\end{aligned}
$$

where $I=\left(I_{1}, I_{2}, \ldots, I_{n}\right)^{T}$. Suppose that $\rho\left(M^{0}\right)<1$. Then, $V_{P_{0}}^{\prime}=0$ if and only if $I=0$. Suppose that $\rho\left(M^{0}\right)=1$. Then, it follows from (20) that $V_{P_{0}}^{\prime}=0$ implies

$$
\sum_{k=1}^{n} \omega_{k}\left[\frac{1}{\delta_{k}+\gamma_{k}} \sum_{j=1}^{n} f_{k j}\left(S_{k}, 0\right) I_{j}(t) Q_{k}\right]=\sum_{k=1}^{n} \omega_{k} I_{k}(t) .
$$

If $S_{k} \neq S_{k}^{0}$, then

$$
\begin{aligned}
\sum_{k=1}^{n} \omega_{k} & {\left[\frac{1}{\delta_{k}+\gamma_{k}} \sum_{j=1}^{n} f_{k j}\left(S_{k}, 0\right) I_{j}(t) Q_{k}\right] } \\
& \leq \sum_{k=1}^{n} \omega_{k}\left[\frac{1}{\delta_{k}+\gamma_{k}} \sum_{j=1}^{n} f_{k j}\left(S_{k}^{0}, 0\right) I_{j}(t) Q_{k}\right] \\
& \leq\left(\omega_{1}, \omega_{2}, \ldots, \omega_{n}\right) M^{0} I \\
& =\left(\omega_{1}, \omega_{2}, \ldots, \omega_{n}\right) \rho\left(M^{0}\right) I \\
& =\left(\omega_{1}, \omega_{2}, \ldots, \omega_{n}\right) I,
\end{aligned}
$$

which implies that (21) has only the trivial solution $I=0$. Therefore, $V_{P_{0}}^{\prime}=0$ if and only if $I_{k}=0$ or $S_{k}=S_{k}^{0}$ provided $\rho\left(M^{0}\right)=1$. It can be verified that the only compact invariant subset of the set where $V_{P_{0}}^{\prime}=0$ is the singleton $\left\{P_{0}\right\}$. By LaSalle's Invariance Principle, $P_{0}$ is globally asymptotically stable in $\Gamma$ if $\rho\left(M^{0}\right) \leq 1$.

If $\mathfrak{R}_{0}>1$ and $I \neq 0$, it is easy to see that

$$
\left[\rho\left(M^{0}\right)-1\right]\left(\omega_{1}, \omega_{2}, \ldots, \omega_{n}\right) I>0 .
$$

It follows from the continuity that $V_{P_{0}}^{\prime}>0$ holds in a small neighborhood of $P_{0}$. This implies that $P_{0}$ is unstable. Using a uniform persistence result from [28] and similar arguments as in $[4,10,13,16,17]$, we know that, if $\Re_{0}>1$, the instability of $P_{0}$ implies the uniform persistence of $(7)$ in $\Gamma$; that is, there exists a positive constant $\epsilon>0$ such that

$$
\liminf _{t \rightarrow \infty} S_{k}(t) \geq \epsilon, \quad \liminf _{t \rightarrow \infty} I_{k}(t) \geq \epsilon, \quad k=1,2, \ldots, n .
$$

The uniform persistence of system (7) together with the uniform boundedness of solutions in $\Gamma$, which follows from the positive invariance of $\Gamma$, implies the existence of an endemic equilibrium $P^{*}$ in $\Gamma$ (see Theorem 2.8 .6 of [29] or Theorem D.3 of [30]). Summarizing the statements above, if $\mathfrak{R}_{0}>1$, system (7) is uniformly persistent and there exists at least one endemic equilibrium $P^{*}$ in $\Gamma$. This completes the proof.

\section{Global Stability of an Endemic Equilibrium}

Denote

$$
H(u)=u-1-\ln u, \quad \forall u>0 .
$$


Obviously, $H: \mathbb{R}^{+} \rightarrow \mathbb{R}^{+}$attains its strict global minimum at $u=1$ and $H(1)=0$.

To get the global stability of $P^{*}$, we make the following assumptions:

$$
\begin{aligned}
& \left(S_{5}\right)\left(\varphi_{k}\left(S_{k}\right)-\varphi_{k}\left(S_{k}^{*}\right)\right)\left(S_{k}-S_{k}^{*}\right) \leq 0 \text { for } S_{k} \geq 0 ; \\
& \left(S_{6}\right)\left(\varphi_{k}\left(S_{k}\right)-\varphi_{k}\left(S_{k}^{*}\right)\right)\left[f_{k k}\left(S_{k}, I_{k}^{*}\right)-f_{k k}\left(S_{k}^{*}, I_{k}^{*}\right)\right]<0 \text { for } \\
& \quad S_{k} \neq S_{k}^{*} ; \\
& \left(S_{7}\right)\left(\left(\left(f_{k k}\left(S_{k}^{*}, I_{k}^{*}\right) f_{k j}\left(S_{k}, I_{j}\right) I_{j}\right) /\left(f_{k k}\left(S_{k}, I_{k}^{*}\right) f_{k j}\left(S_{k}^{*}, I_{j}^{*}\right) I_{j}^{*}\right)\right)\right. \\
& \quad-1)\left(1-\left(\left(f_{k k}\left(S_{k}, I_{k}^{*}\right) f_{k j}\left(S_{k}^{*}, I_{j}^{*}\right)\right) /\left(f _ { k k } ( S _ { k } ^ { * } , I _ { k } ^ { * } ) f _ { k j } \left(S_{k},\right.\right.\right.\right. \\
& \left.\left.\left.\left.\quad I_{j}\right)\right)\right)\right) \leq 0 \text { for } S_{k}, I_{j}>0 .
\end{aligned}
$$

Theorem 3. Assume that the functions $\varphi_{k}$ and $f_{k j}$ satisfy $\left(S_{1}\right)-\left(S_{7}\right)$, and the matrix $M^{0}$ is irreducible. If $\mathfrak{R}_{0}>1$, then there is a unique endemic equilibrium $P^{*}$ for system (12), and $P^{*}$ is globally asymptotically stable in the interior of $\Gamma$.

Proof. Define a Lyapunov functional as

$$
\begin{aligned}
V_{P^{*}}= & Q_{k} \int_{S_{k}^{*}}^{S_{k}(t)} \frac{f_{k k}\left(\eta, I_{k}^{*}\right)-f_{k k}\left(S_{k}^{*}, I_{k}^{*}\right)}{f_{k k}\left(\eta, I_{k}^{*}\right)} \mathrm{d} \eta \\
& +I_{k}^{*} H\left(\frac{I_{k}(t)}{I_{k}^{*}}\right)+V_{+},
\end{aligned}
$$

where

$$
\begin{aligned}
V_{+}=\sum_{j=1}^{n} \int_{0}^{\infty} f_{k j}\left(S_{k}^{*}, I_{j}^{*}\right) I_{j}^{*} J(u) \\
\quad \times H\left(\frac{f_{k j}\left(S_{k}(t-u), I_{j}(t-u)\right) I_{j}(t-u)}{f_{k j}\left(S_{k}^{*}, I_{j}^{*}\right) I_{j}^{*}}\right) \mathrm{d} u .
\end{aligned}
$$

First, we calculate the derivative of $V_{+}$; then, we have

$V_{+}^{\prime}$

$$
\begin{aligned}
&=\sum_{j=1}^{n} \int_{0}^{\infty} f_{k j}\left(S_{k}^{*}, I_{j}^{*}\right) I_{j}^{*} J(u) \frac{\mathrm{d}}{\mathrm{d} t} \\
& \times H\left(\frac{f_{k j}\left(S_{k}(t-u), I_{j}(t-u)\right) I_{j}(t-u)}{f_{k j}\left(S_{k}^{*}, I_{j}^{*}\right) I_{j}^{*}}\right) \mathrm{d} u \\
&=-\sum_{j=1}^{n} \int_{0}^{\infty} f_{k j}\left(S_{k}^{*}, I_{j}^{*}\right) I_{j}^{*} J(u) \frac{\mathrm{d}}{\mathrm{d} u} \\
& \quad \times H\left(\frac{f_{k j}\left(S_{k}(t-u), I_{j}(t-u)\right) I_{j}(t-u)}{f_{k j}\left(S_{k}^{*}, I_{j}^{*}\right) I_{j}^{*}}\right) \mathrm{d} u
\end{aligned}
$$

$$
\begin{aligned}
= & -\sum_{j=1}^{n} f_{k j}\left(S_{k}^{*}, I_{j}^{*}\right) I_{j}^{*} J(u) \\
& \times\left. H\left(\frac{f_{k j}\left(S_{k}(t-u), I_{j}(t-u)\right) I_{j}(t-u)}{f_{k j}\left(S_{k}^{*}, I_{j}^{*}\right) I_{j}^{*}}\right)\right|_{u=0} ^{\infty}
\end{aligned}
$$

$$
\begin{aligned}
& +\sum_{j=1}^{n} \int_{0}^{\infty} f_{k j}\left(S_{k}^{*}, I_{j}^{*}\right) I_{j}^{*} \\
& \times H\left(\frac{f_{k j}\left(S_{k}(t-u), I_{j}(t-u)\right) I_{j}(t-u)}{f_{k j}\left(S_{k}^{*}, I_{j}^{*}\right) I_{j}^{*}}\right) \mathrm{d} J(u) \\
& =\sum_{j=1}^{n} Q_{k} f_{k j}\left(S_{k}^{*}, I_{j}^{*}\right) I_{j}^{*} H\left(\frac{f_{k j}\left(S_{k}(t), I_{j}(t)\right) I_{j}(t)}{f_{k j}\left(S_{k}^{*}, I_{j}^{*}\right) I_{j}^{*}}\right) \\
& -\sum_{j=1}^{n} \int_{0}^{\infty} f_{k j}\left(S_{k}^{*}, I_{j}^{*}\right) I_{j}^{*} g_{k}(u) e^{-\delta_{k} u} \\
& \times H\left(\frac{f_{k j}\left(S_{k}(t-u), I_{j}(t-u)\right) I_{j}(t-u)}{f_{k j}\left(S_{k}^{*}, I_{j}^{*}\right) I_{j}^{*}}\right) \mathrm{d} u \\
& =\sum_{j=1}^{n} Q_{k}\left(f_{k j}\left(S_{k}(t), I_{j}(t)\right) I_{j}(t)-f_{k j}\left(S_{k}^{*}, I_{j}^{*}\right) I_{j}^{*}\right. \\
& \left.\times \ln \frac{f_{k j}\left(S_{k}(t), I_{j}(t)\right) I_{j}(t)}{f_{k j}\left(S_{k}^{*}, I_{j}^{*}\right) I_{j}^{*}}\right) \\
& -\sum_{j=1}^{n} \int_{0}^{\infty} g_{k}(u) e^{-\delta_{k} u} \\
& \times\left[f_{k j}\left(S_{k}(t-u), I_{j}(t-u)\right) I_{j}(t-u)\right. \\
& -f_{k j}\left(S_{k}^{*}, I_{j}^{*}\right) I_{j}^{*} . \\
& \left.\times \ln \frac{f_{k j}\left(S_{k}(t-u), I_{j}(t-u)\right) I_{j}(t-u)}{f_{k j}\left(S_{k}^{*}, I_{j}^{*}\right) I_{j}^{*}}\right] \mathrm{d} u .
\end{aligned}
$$

Calculating the time derivative of $V_{P^{*}}$ along the solution of system (12), we have

$$
\begin{aligned}
V_{P^{*}}^{\prime}= & Q_{k}\left(1-\frac{f_{k k}\left(S_{k}^{*}, I_{k}^{*}\right)}{f_{k k}\left(S_{k}(t), I_{k}^{*}\right)}\right) \\
\times & {\left[\varphi_{k}\left(S_{k}(t)\right)-\sum_{j=1}^{n} f_{k j}\left(S_{k}(t), I_{j}(t)\right) I_{j}(t)\right] } \\
+ & \left(1-\frac{I_{k}^{*}}{I_{k}(t)}\right) \\
\times & {\left[\sum _ { j = 1 } ^ { n } \int _ { 0 } ^ { \infty } f _ { k j } \left(S_{k}(u),\right.\right.} \\
& \left.\quad-\left(\delta_{k}+\gamma_{k}\right) I_{k}(t)\right]+V_{+}^{\prime} .
\end{aligned}
$$


6

Abstract and Applied Analysis

Using equilibrium equations (16), we have

$$
\begin{aligned}
& V_{P^{*}}^{\prime}= Q_{k}\left(\varphi_{k}\left(S_{k}(t)\right)-\varphi_{k}\left(S_{k}^{*}\right)\right)\left(1-\frac{f_{k k}\left(S_{k}^{*}, I_{k}^{*}\right)}{f_{k k}\left(S_{k}(t), I_{k}^{*}\right)}\right) \\
&+\sum_{j=1}^{n} Q_{k} f_{k j}\left(S_{k}^{*}, I_{j}^{*}\right) I_{j}^{*}-\sum_{j=1}^{n} Q_{k} f_{k j}\left(S_{k}(t), I_{j}(t)\right) I_{j}(t) \\
&-\sum_{j=1}^{n} Q_{k} f_{k j}\left(S_{k}^{*}, I_{j}^{*}\right) I_{j}^{*} \frac{f_{k k}\left(S_{k}^{*}, I_{k}^{*}\right)}{f_{k k}\left(S_{k}(t), I_{k}^{*}\right)} \\
&+\sum_{j=1}^{n} Q_{k} f_{k j}\left(S_{k}(t), I_{j}(t)\right) I_{j}(t) \frac{f_{k k}\left(S_{k}^{*}, I_{k}^{*}\right)}{f_{k k}\left(S_{k}(t), I_{k}^{*}\right)} \\
&+\sum_{j=1}^{n} \int_{0}^{\infty} f_{k j}\left(S_{k}(t-u), I_{j}(t-u)\right) \\
&-\frac{I_{k}(t)}{I_{k}^{*}} \sum_{j=1}^{n} \int_{0}^{\infty} f_{k j}\left(S_{k}^{*}, I_{j}^{*}\right) I_{j}^{*} e^{-\delta_{k} u} g_{k}(u) \mathrm{d} u \\
&-\frac{I_{k}^{*}}{I_{k}(t)} \sum_{j=1}^{n} \int_{0}^{\infty} f_{k j}\left(S_{k}(t-u), I_{j}(t-u)\right) \\
&+\sum_{j=1}^{n} \int_{0}^{\infty} f_{k j}\left(S_{k}^{*}, I_{j}^{*}\right) I_{j}^{*} e^{-\delta_{k} u} g_{k}(u) \mathrm{d} u+V_{+}^{\prime} \cdot \\
& \times I_{j}(t-u) e^{-\delta_{k} u} g_{k}(u) \mathrm{d} u \\
& \mathrm{~d} u
\end{aligned}
$$

(30)

Using $V_{+}^{\prime}$, we rewrite (30) as

$$
\begin{array}{r}
V_{P^{*}}^{\prime}=Q_{k}\left(\varphi_{k}\left(S_{k}(t)\right)-\varphi_{k}\left(S_{k}^{*}\right)\right)\left(1-\frac{f_{k k}\left(S_{k}^{*}, I_{k}^{*}\right)}{f_{k k}\left(S_{k}(t), I_{k}^{*}\right)}\right) \\
+\sum_{j=1}^{n} Q_{k} f_{k j}\left(S_{k}^{*}, I_{j}^{*}\right) I_{j}^{*} \\
\times\left[2-\frac{f_{k k}\left(S_{k}^{*}, I_{k}^{*}\right)}{f_{k k}\left(S_{k}(t), I_{k}^{*}\right)}\right. \\
+\frac{f_{k k}\left(S_{k}^{*}, I_{k}^{*}\right) f_{k j}\left(S_{k}(t), I_{j}(t)\right) I_{j}(t)}{f_{k k}\left(S_{k}(t), I_{k}^{*}\right) f_{k j}\left(S_{k}^{*}, I_{j}^{*}\right) I_{j}^{*}} \\
-\sum_{j=1}^{n} \int_{0}^{\infty} f_{k j}\left(S_{k}^{*}, I_{j}^{*}\right) I_{j}^{*} g_{k}(u) e^{-\delta_{k} u}
\end{array}
$$

$$
\begin{aligned}
& {\left[\frac{I_{k}^{*} f_{k j}\left(S_{k}(t-u), I_{j}(t-u)\right) I_{j}(t-u)}{I_{k}(t) f_{k j}\left(S_{k}^{*}, I_{j}^{*}\right) I_{j}^{*}}\right.} \\
& \left.\quad-\ln \frac{f_{k j}\left(S_{k}(t-u), I_{j}(t-u)\right) I_{j}(t-u)}{f_{k j}\left(S_{k}(t), I_{j}(t)\right) I_{j}(t)}\right] \mathrm{d} u .
\end{aligned}
$$

Therefore,

$V_{P^{*}}^{\prime}$

$$
\begin{aligned}
& =Q_{k}\left(\varphi_{k}\left(S_{k}(t)\right)-\varphi_{k}\left(S_{k}^{*}\right)\right)\left(1-\frac{f_{k k}\left(S_{k}^{*}, I_{k}^{*}\right)}{f_{k k}\left(S_{k}(t), I_{k}^{*}\right)}\right) \\
& -\sum_{j=1}^{n} Q_{k} f_{k j}\left(S_{k}^{*}, I_{j}^{*}\right) I_{j}^{*} \\
& \times\left[H\left(\frac{f_{k k}\left(S_{k}^{*}, I_{k}^{*}\right)}{f_{k k}\left(S_{k}(t), I_{k}^{*}\right)}\right)\right. \\
& \left.+H\left(\frac{f_{k k}\left(S_{k}(t), I_{k}^{*}\right) f_{k j}\left(S_{k}^{*}, I_{j}^{*}\right)}{f_{k k}\left(S_{k}^{*}, I_{k}^{*}\right) f_{k j}\left(S_{k}(t), I_{j}(t)\right)}\right)\right] \\
& +\sum_{j=1}^{n} Q_{k} f_{k j}\left(S_{k}^{*}, I_{j}^{*}\right) \\
& \times\left(\frac{f_{k k}\left(S_{k}^{*}, I_{k}^{*}\right) f_{k j}\left(S_{k}(t), I_{j}(t)\right) I_{j}(t)}{f_{k k}\left(S_{k}(t), I_{k}^{*}\right) f_{k j}\left(S_{k}^{*}, I_{j}^{*}\right) I_{j}^{*}}-1\right) \\
& \times\left(1-\frac{f_{k k}\left(S_{k}(t), I_{k}^{*}\right) f_{k j}\left(S_{k}^{*}, I_{j}^{*}\right)}{f_{k k}\left(S_{k}^{*}, I_{k}^{*}\right) f_{k j}\left(S_{k}(t), I_{j}(t)\right)}\right) \\
& -\sum_{j=1}^{n} \int_{0}^{\infty} f_{k j}\left(S_{k}^{*}, I_{j}^{*}\right) I_{j}^{*} g_{k}(u) e^{-\delta_{k} u} \\
& \times H\left(\frac{I_{k}^{*} f_{k j}\left(S_{k}(t-u), I_{j}(t-u)\right) I_{j}(t-u)}{I_{k}(t) f_{k j}\left(S_{k}^{*}, I_{j}^{*}\right) I_{j}^{*}}\right) \mathrm{d} u \\
& +\sum_{j=1}^{n} Q_{k} f_{k j}\left(S_{k}^{*}, I_{j}^{*}\right) \\
& \times I_{j}^{*}\left[\frac{I_{j}(t)}{I_{j}^{*}}-\frac{I_{k}(t)}{I_{k}^{*}}-\ln \frac{I_{j}(t)}{I_{j}^{*}}+\ln \frac{I_{k}(t)}{I_{k}^{*}}\right] .
\end{aligned}
$$

Furthermore, under $\left(S_{5}\right)-\left(S_{7}\right)$, we have

$$
\begin{aligned}
V_{P^{*}}^{\prime} \leq \sum_{j=1}^{n} Q_{k} f_{k j}\left(S_{k}^{*}, I_{j}^{*}\right) \\
\quad \times I_{j}^{*}\left[\frac{I_{j}(t)}{I_{j}^{*}}-\frac{I_{k}(t)}{I_{k}^{*}}-\ln \frac{I_{j}(t)}{I_{j}^{*}}+\ln \frac{I_{k}(t)}{I_{k}^{*}}\right] .
\end{aligned}
$$


Obviously, the equalities in (33) hold if and only if $S_{k}=S_{k}^{*}$ and $I_{k}=I_{k}^{*}, k=1,2, \ldots, n$. Therefore, the functional $V=$ $\sum_{k=1}^{n} v_{k} V_{P^{*}}$ as defined in Theorem 3.1 of [12] is a Lyapunov function for system (12). Using similar arguments as in [4, $8-13,16,17]$, one can show that the largest invariant subset where $V_{p^{*}}^{\prime}=0$ is the singleton $\left\{P^{*}\right\}$. By LaSalle's Invariance Principle, $P^{*}$ is globally asymptotically stable in the interior of $\Gamma$. This completes the proof of Theorem 3 .

\section{Conflict of Interests}

The authors declare that there is no conflict of interests regarding the publication of this paper.

\section{Acknowledgments}

The authors would like to thank the anonymous referees and the editor for very helpful suggestions and comments which led to improvements of our original paper. J. Wang is supported by National Natural Science Foundation of China (no. 11201128), the Science and Technology Research Project of the Department of Education of Heilongjiang Province (no. 12531495), the Natural Science Foundation of Heilongjiang Province (no. A201211), and the Science and Technology Innovation Team in Higher Education Institutions of Heilongjiang Province.

\section{References}

[1] R. M. Anderson and R. M. May, "Population biology of infectious diseases I," Nature, vol. 280, pp. 361-367, 1979.

[2] S. J. Gao, Z. D. Teng, and D. H. Xie, "The effects of pulse vaccination on SEIR model with two time delays," Applied Mathematics and Computation, vol. 201, no. 1-2, pp. 282-292, 2008.

[3] A. Korobeinikov, "Global properties of SIR and SEIR epidemic models with multiple parallel infectious stages," Bulletin of Mathematical Biology, vol. 71, no. 1, pp. 75-83, 2009.

[4] M. Y. Li, J. R. Graef, L. Wang, and J. Karsai, "Global dynamics of a SEIR model with varying total population size," Mathematical Biosciences, vol. 160, no. 2, pp. 191-213, 1999.

[5] P. van den Driessche, L. Wang, and X. Zou, "Modeling diseases with latency and relapse," Mathematical Biosciences and Engineering, vol. 4, no. 2, pp. 205-219, 2007.

[6] P. van den Driessche and X. Zou, "Modeling relapse in infectious diseases," Mathematical Biosciences, vol. 207, no. 1, pp. 89103, 2007.

[7] S. Liu, S. Wang, and L. Wang, "Global dynamics of delay epidemic models with nonlinear incidence rate and relapse," Nonlinear Analysis. Real World Applications, vol. 12, no. 1, pp. 119-127, 2011.

[8] H. Guo, M. Y. Li, and Z. Shuai, "Global stability of the endemic equilibrium of multigroup SIR epidemic models," Canadian Applied Mathematics Quarterly, vol. 14, no. 3, pp. 259-284, 2006.

[9] H. Guo, M. Y. Li, and Z. Shuai, "A graph-theoretic approach to the method of global Lyapunov functions," Proceedings of the American Mathematical Society, vol. 136, no. 8, pp. 2793-2802, 2008.
[10] T. Kuniya, "Global stability of a multi-group SVIR epidemic model," Nonlinear Analysis. Real World Applications, vol. 14, no. 2, pp. 1135-1143, 2013.

[11] M. Y. Li, Z. S. Shuai, and C. C. Wang, "Global stability of multi-group epidemic models with distributed delays," Journal of Mathematical Analysis and Applications, vol. 361, no. 1, pp. $38-47,2010$.

[12] M. Y. Li and Z. S. Shuai, "Global-stability problem for coupled systems of differential equations on networks," Journal of Differential Equations, vol. 248, no. 1, pp. 1-20, 2010.

[13] R. Sun, "Global stability of the endemic equilibrium of multigroup SIR models with nonlinear incidence," Computers \& Mathematics with Applications, vol. 60, no. 8, pp. 2286-2291, 2010.

[14] R. Sun and J. Shi, "Global stability of multigroup epidemic model with group mixing and nonlinear incidence rates," Applied Mathematics and Computation, vol. 218, no. 2, pp. 280286, 2011.

[15] H. Shu, D. Fan, and J. Wei, "Global stability of multi-group SEIR epidemic models with distributed delays and nonlinear transmission," Nonlinear Analysis. Real World Applications, vol. 13, no. 4, pp. 1581-1592, 2012.

[16] J. Wang, J. Zu, X. Liu, G. Huang, and J. Zhang, "Global dynamics of a multi-group epidemic model with general relapse distribution and nonlinear incidence rate," Journal of Biological Systems, vol. 20, no. 3, pp. 235-258, 2012.

[17] J. Wang, Y. Takeuchi, and S. Liu, "A multi-group SVEIR epidemic model with distributed delay and vaccination," International Journal of Biomathematics, vol. 5, no. 3, Article ID 1260001, 2012.

[18] L. Zhang, J. Pang, and J. Wang, "Stability analysis of a multigroup epidemic model with general exposed distribution and nonlinear incidence rates," Abstract and Applied Analysis, vol. 2013, Article ID 354287, 11 pages, 2013.

[19] K. Hattaf, A. A. Lashari, Y. Louartassi, and N. Yousfi, "A delayed SIR epidemic model with general incidence rate," Electronic Journal of Qualitative Theory of Differential Equations, vol. 3, pp. $1-9,2013$

[20] P. Georgescu, Y.-H. Hsieh, and H. Zhang, "A Lyapunov functional for a stage-structured predator-prey model with nonlinear predation rate," Nonlinear Analysis. Real World Applications, vol. 11, no. 5, pp. 3653-3665, 2010.

[21] X. Wang, Y. D. Tao, and X. Y. Song, "Pulse vaccination on SEIR epidemic model with nonlinear incidence rate," Applied Mathematics and Computation, vol. 210, no. 2, pp. 398-404, 2009.

[22] R. K. Miller, Nonlinear Volterra Integral Equations, W. A. Benjamin, New York, NY, USA, 1971.

[23] J. K. Hale and S. M. Verduyn Lunel, Introduction to FunctionalDifferential Equations, vol. 99 of Applied Mathematical Sciences, Springer, New York, NY, USA, 1993.

[24] Y. Hino, S. Murakami, and T. Naito, Functional-Differential Equations with Infinite Delay, vol. 1473 of Lecture Notes in Mathematics, Springer, 1991.

[25] O. Diekmann, J. A. P. Heesterbeek, and J. A. J. Metz, "On the definition and the computation of the basic reproduction ratio $R_{0}$ in models for infectious diseases in heterogeneous populations," Journal of Mathematical Biology, vol. 28, no. 4, pp. 365-382, 1990. 
[26] P. van den Driessche and J. Watmough, "Reproduction numbers and sub-threshold endemic equilibria for compartmental models of disease transmission," Mathematical Biosciences, vol. 180, pp. 29-48, 2002.

[27] A. Berman and R. J. Plemmons, Nonnegative Matrices in the Mathematical Sciences, Academic Press, New York, NY, USA, 1979.

[28] H. I. Freedman, S. G. Ruan, and M. X. Tang, "Uniform persistence and flows near a closed positively invariant set," Journal of Dynamics and Differential Equations, vol. 6, no. 4, pp. 583-600, 1994.

[29] N. P. Bhatia and G. P. Szegő, Dynamical Systems: Stability Theory and Applications, Springer, Berlin, Germany, 1967.

[30] H. L. Smith and P. Waltman, The Theory of the Chemostat, Cambridge University Press, 1995. 


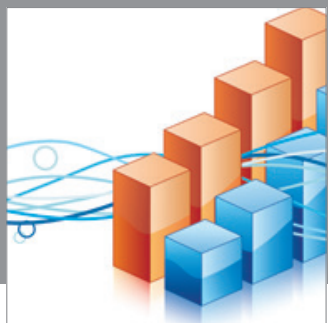

Advances in

Operations Research

mansans

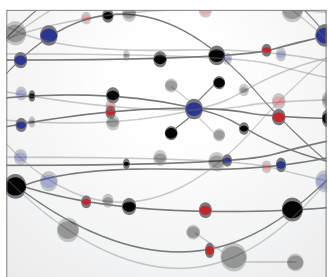

The Scientific World Journal
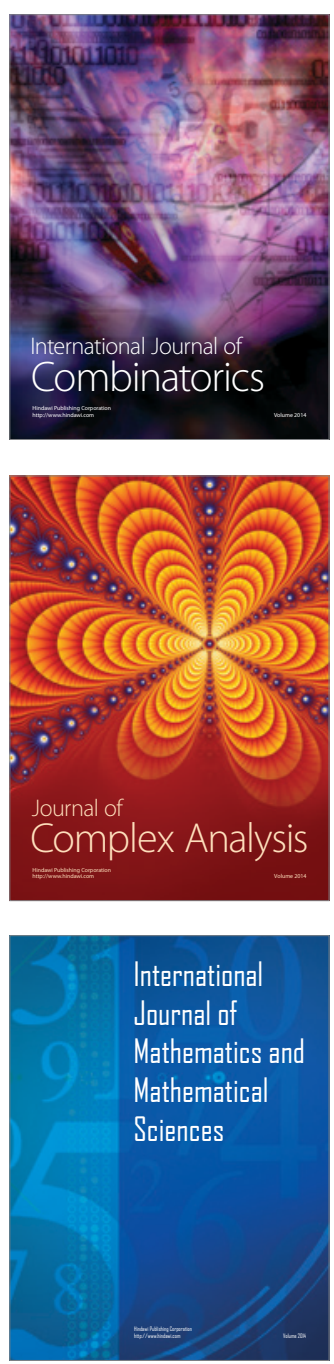
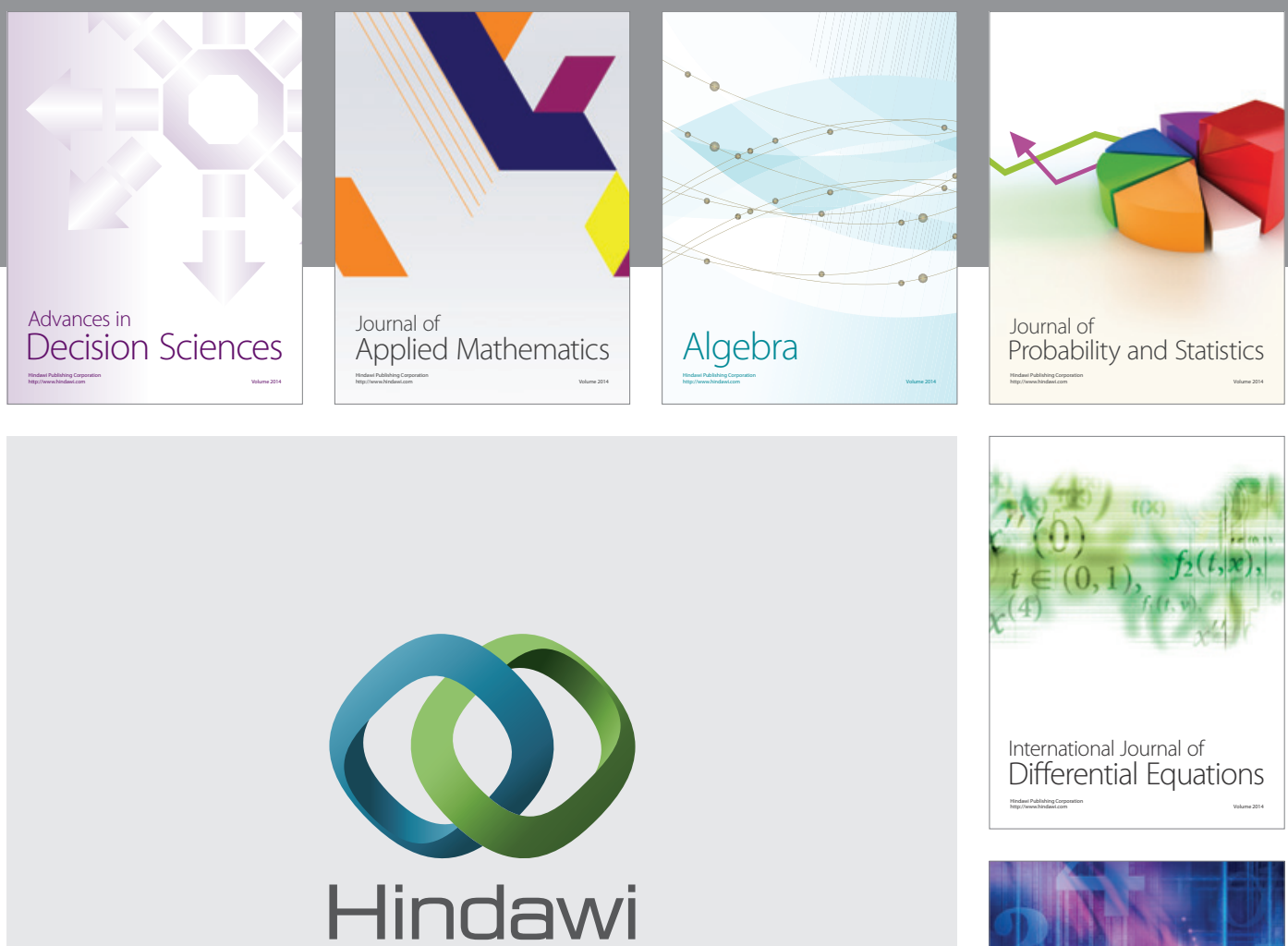

Submit your manuscripts at http://www.hindawi.com
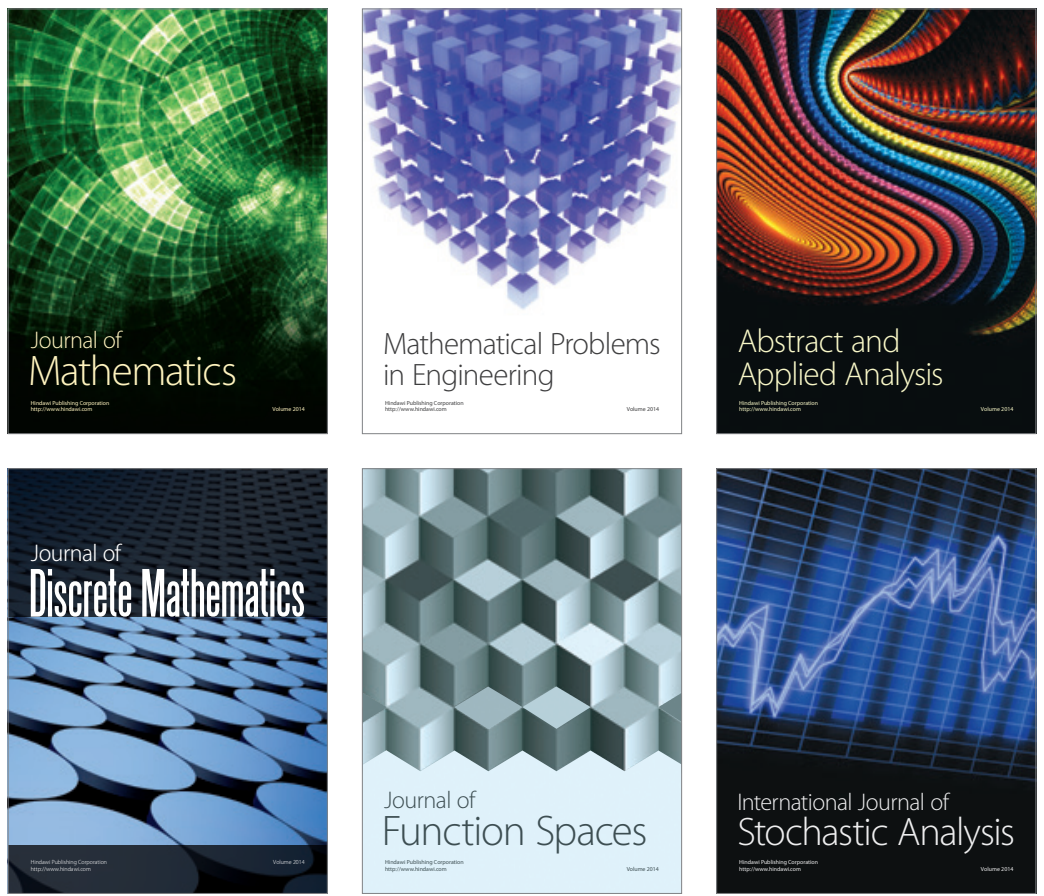

Journal of

Function Spaces

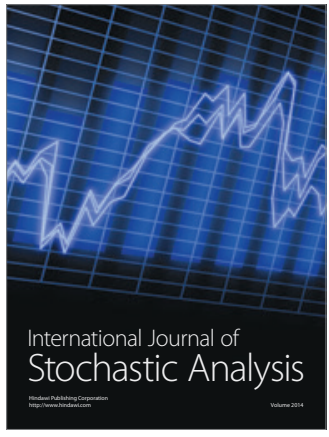

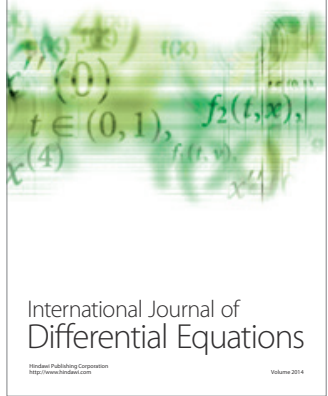
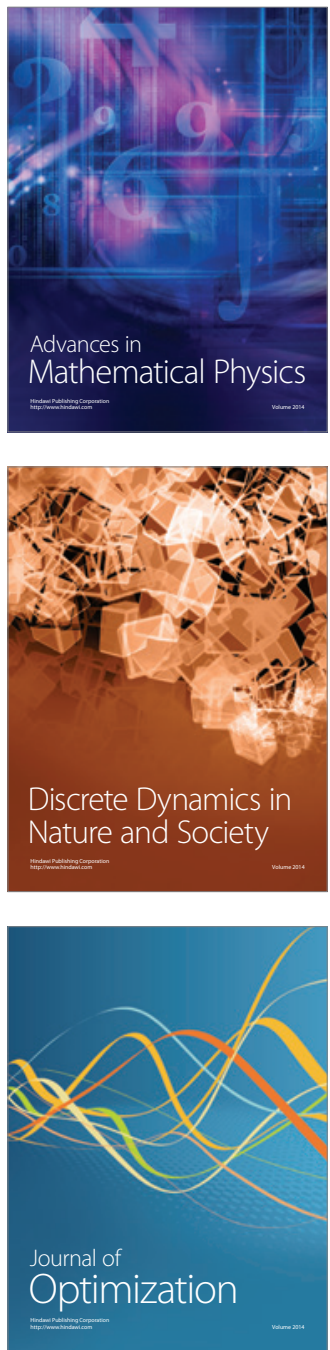